\title{
Persistence of statin treatment - the impact of analytic method when estimating drug survival
}

\author{
Francisco Oteiza $^{\mathrm{a}}$, Hanna Isabel Løyland ${ }^{\mathrm{a}}$, Christoffer Bugge ${ }^{\mathrm{a}, \mathrm{b}}$, Ivar Sønbø Kristiansen ${ }^{\mathrm{a}, \mathrm{b}, \mathrm{c}}$ \\ and Henrik Støvring ${ }^{\mathrm{d}}$ \\ a) Oslo Economics, Oslo, Norway \\ b) Department of Health Management and Health Economics, University of Oslo, Norway \\ c) Department of Public Health, University of Southern Denmark, Odense, Denmark \\ d) Department of Public Health, University of Aarhus, Aarhus, Denmark \\ Correspondence: i.s.kristiansen@medisin.uio.no
}

\begin{abstract}
Background: There is ample evidence for several pharmaceutical treatments that adherence in terms of treatment duration and dose is suboptimal. The actual drug intake cannot be observed directly in prescription databases, which only register drug redeemed and a limited number of patient characteristics. Consequently, the actual dose and duration of treatment must be inferred from observed redemptions. Persistence can then be expressed as treatment duration (also referred to as drug survival).

Method: We used data from the Norwegian Prescription Database (NorPD) on redemptions of statins (ATCcode C10AA) for the period 2010-2019 to explore three methods for determining prescription durations and in turn persistence (treatment duration): (i) The DDD-method using the number of DDD redeemed; (ii) The dose-unit approach using the number of tablets redeemed; (iii) The reverse waiting time distribution method (WTD), which estimates prescription duration as the $90^{\text {th }}$ percentile of the distribution within which patients in ongoing treatment will have a new subsequent redemption. The three methods for estimating prescription duration were then used to estimate treatment duration using Kaplan Meier (KM) survival functions. For the DDD-method and the dose-unit approach we conducted sensitivity analyses assuming that one DDD or one tablet would last for $1.00,1.25$ or 2.00 days. We also tested the impact of grace periods in sensitivity analyses. Results: Treatment duration and drug survival varied substantially for the same patients depending on the chosen method, duration of a DDD or a tablet, and inclusion of grace periods. The $25^{\text {th }}$ percentile of treatment duration was 100 days for the DDD approach with one DDD per day, 100 days with the dose-unit approach with one tablet per day and 453 days with the WTD approach.

Conclusion: When estimating treatment duration from prescription databases one should be aware that these measures of persistence are highly influenced by the chosen methodology. The choice of method should be informed by the clinical context with a preference for use of methods based on a formal model.
\end{abstract}

This is an open access article distributed under the Creative Commons Attribution Licence, which permits unrestricted use, distribution, and reproduction in any medium, provided the original work is properly cited.

\section{BACKGROUND}

Hypercholesterolaemia is an important risk factor for atherosclerotic cardiovascular disease (ASCVD). HMG$\mathrm{CoA}$ reductase inhibitors (statins) represent a class of lipid-lowering medications that reduce the liver synthesis of cholesterol. Statins reduce ACVD morbidity and mortality, but should be used continuously and lifelong to achieve the maximum benefit [1].

Previous studies demonstrate that the duration of statin treatment is often not lifelong as recommended in guidelines. In practice, many patients reduce the daily dose if the tablet may be split, take tablets less frequently than every day, or have breaks lasting weeks or months. A systematic review with 84 real world studies concluded that good statin adherence is associated with reductions in cardiovascular events and mortality [2]. In another systematic review, 28 observational studies consistently reported increased risk of CVD and mortality among non-adherent and non-persistent individuals [3].

Adherence has been defined as "the process by which patients take their medications as prescribed" [4,5]. It has three components: initiation, implementation, and discontinuation. Persistence is defined as the "time from initiation to discontinuation" and represents a quantitative concept [5]. In this paper, we explore methods for estimating persistence using statins as case. [6] Statin tablets are offered in different strengths, most of them ranging from 10 to $80 \mathrm{mg}$. This adds complications to studies of persistence based on dispensing volumes and dates, where actual intake, and thus the duration of treatment, after a dispensing is unobserved.

In this study, we adopt three different strategies to estimate prescription duration:

- Defined Daily Dose (DDD) assigning duration to each dispensing (system defined duration)

- Dose-unit assuming that patients take one tablet per day (simple empirical definition of duration)

- Reverse Waiting Time Distribution (WTD) to estimate duration of each dispensing (data driven definition based on actual usage)

When prescription durations have been estimated, sequences of prescriptions can be joined together into 
treatment episodes defined as prescriptions without treatment breaks in between, possibly allowing for a grace period.

The first two of the approaches for determining prescription durations are decision rules, which largely reflect how patients are intended to take the medication. The DDD is defined by experts appointed by the WHO Collaborating Centre for Drug Statistics Methodology as "...the assumed average maintenance dose per day for a drug used for its main indication in adults" [7]. According to their summary of product characteristics (SPC), statins marketed in Norway are intended to be taken as one pill per day, why this also would appear to be a reasonable choice. By contrast, the reverse WTD relies on actual dispensing and usage patterns to determine duration of a dispensing. It is therefore an alternative method, which is data driven. It could therefore potentially aid in identifying discrepancies between actual and intended usage. The reverse WTD has been validated in simulation studies and empirically for warfarin treatment $[8,9]$.

The aim of this study was to compare the three strategies when applied to estimate persistence of statin treatment. We will further investigate how the total amount of dispensed statin over time compares to treatment duration as a measure of persistence.

\section{METHODS}

We received data from the Norwegian Prescription Database (NorPD) for all redemptions of statins (ATC code C10AA) for the period 2010-2019. For each redemption we received information on the date of redemption, number of DDD redeemed, tablet strengths (mg), and number of tablets dispensed. We also received information on patients' age, year of birth and date of death, if applicable. Each patient had an anonymous patient identification number, which allowed us to follow patients over time.

Before conducting our analysis, we performed data quality checks and found a small number of errors in the DDD values. Seven redemptions had negative DDD values, while three others had more than 2,400 DDDs, which we considered to be above a realistic value for a single prescription. These prescriptions had been filled by eight different individuals. We thus excluded these eight individuals from our sample, resulting in a total of 410 omitted observations.

This left a total of almost 21 million redemptions, prescribed to 886,496 unique individuals, between 2010 and 2019 in all of Norway. To ensure that each firsttime redemption was really the first for each patient, and not just the first observed redemption in the dataset, we used 2010-2011 as a washout period. In other words, we removed all patients from our sample who had a redemption before January $1^{\text {st }}$, 2012, a total of 17 million prescriptions and 530,857 unique individuals. This left us with only those patients whose first redemption fell on that day or later, a total of 355,639 unique patients and more than four million prescriptions during the 2012-2019 period.

We then produced a series of descriptive statistics, including patient demographics, number of unique patients and number of prescriptions by drug, over the course of the years in our sample. We also calculated the average number of DDDs prescribed to each patient per year and by year.

For the rest of the analysis, we reduced the sample size to allow for more efficient computer processing. We did this by randomly sampling $25 \%$ of the individuals and omitting all prescriptions that did not belong to these patients. This left us with 88,913 unique individuals, and 1,091,792 prescriptions over the study period. Our estimates of persistence (drug survival) were all based on this representative dataset, with negligible impact on the precision of our estimates, as evidenced by the narrow confidence intervals.

In analyses based on decision rules, we assumed that each tablet or DDD dispensed could last for 1.00, 1.25 or 2.00 days, which allowed us to estimate the amount of time after each prescription for which a patient was considered treated (prescription duration). Since patients are prescribed varying daily statin doses based on the prescribers' practice and the patients' lipid levels and treatment responses, patients could be prescribed tablets with a statin content, which was lower, higher, or equal to the theoretical DDD of the drug. Therefore, we also estimated persistence by assuming that the daily prescribed dose in mg would last $1.00,1.25$ or 2.00 days. NorPd does not hold information on the prescribed dose, so here we assumed that the dose was equal to one tablet per day.

We joined together prescription durations into treatment episodes whenever a new dispensing was observed for a patient while still in treatment with medication from the previous dispensing. We allowed for stockpiling by adding any unused medication (according to our definition of use) to the new dispensing. Finally, in a sensitivity analysis we added "grace periods" of 15 and 30 days after the end of a prescription duration, i.e. we considered treatment to have continued if a new dispensing occurred within the grace period.

In addition to analyses based on decision rules, we also used the reverse waiting time distribution (WTD) to estimate prescription duration [10]. We examined whether WTD estimates for different calendar years varied due to changes in usage patterns. Based on the obtained parameter estimates we then assigned the average estimated $90^{\text {th }}$ percentile as the duration for all prescriptions observed across the 2012-2019 period. Then, as we did in the DDD and dose-unit approaches, we joined prescription periods to create uninterrupted treatment episodes. Again, in a sensitivity analysis we considered grace periods.

For the first treatment episode of each individual as constructed above, we report their median and $10^{\text {th }}$ and $25^{\text {th }}$ percentile duration as measures of persistence. In a sub-analysis, we report the corresponding measures 
Table 1. Summary statistics for patients who started on a new course of statin treatment by year*.

\begin{tabular}{|c|c|c|c|c|c|c|c|c|c|}
\hline Descriptive statistic & 2012 & 2013 & 2014 & 2015 & 2016 & 2017 & 2018 & 2019 & Total \\
\hline Number of new patients & 48,500 & 42,367 & 40,848 & 43,183 & 46,855 & 52,507 & 51,540 & 51,363 & 377,163 \\
\hline Number of existing patients & 0 & 37,518 & 66,729 & 92,870 & 118,381 & 145,442 & 176,531 & 202,449 & 839,920 \\
\hline Number of patients deceased & 355 & 1,006 & 1,528 & 2,076 & 2,838 & 3,409 & 4,147 & 4,849 & 20,208 \\
\hline Number of living patients & 48,145 & 78,879 & 106,049 & 133,977 & 162,398 & 194,540 & 223,924 & 248,963 & $1,196,875$ \\
\hline Mean patient age & 60.9 & 61.5 & 62.0 & 62.3 & 62.6 & 62.9 & 63.3 & 63.66 & 62.77 \\
\hline Proportion female $(\%)$ & $45 \%$ & $45 \%$ & $44 \%$ & $44 \%$ & $44 \%$ & $44 \%$ & $43 \%$ & $43 \%$ & $44 \%$ \\
\hline Total number of redemptions & 121,265 & 250,710 & 366,953 & 479,675 & 598,598 & 730,779 & 868,197 & 968,131 & $4,384,308$ \\
\hline $\begin{array}{l}\text { Mean number of DDDs per } \\
\text { patient per year } \\
\text { Mean tablet strength (mg) among }\end{array}$ & 272 & 339 & 371 & 389 & 400 & 405 & 417 & 427 & 397 \\
\hline all redemptions in the year & 30.21 & 30.55 & 30.72 & 30.76 & 30.58 & 30.33 & 30.18 & 30.19 & 30.39 \\
\hline
\end{tabular}

* The number of new patients per year represents the number of unique patient IDs observed each year, for patients who have not redeemed another prescription during the previous two years. Patients with a gap between prescriptions of under two years are counted as existing patients. The total number of unique patients over the whole sample is 355,639 of whom 335,431 were still alive by the end of 2019 .

when all treatment episodes are included (see Appendix). We supplemented the analysis with Kaplan-Meier curves of drug survival stratified by sex and age category. In our primary analysis, we only considered the first treatment episode to avoid downward bias of estimated drug survival, which would occur if users had been included with all their observed treatment episodes - to have multiple episodes observed for the same user, these will by necessity have to be comparatively short.

\section{RESULTS}

The full analysis dataset included 355,639 patients among whom $44 \%$ were female. The proportion of females declined from $45 \%$ to $43 \%$ during the study period. The mean age of those dispensed a statin in 2019 was 63.7 years. That year, seven different statins were dispensed to these patients, a total 968,131 times (Table 1).

The number of unique patients in our dataset is presented in the following way. After the wash-out period, each patient is recorded as a new patient in the dataset if no earlier prescriptions are observed for a period of two years. Patients who begin treatment but have less than two years since their last prescription, are recorded as existing patients. Finally, the number of patients who die is counted each year. This leads us to the number of living patients observed each year. This number increases over time, as the rate at which new patients begin their treatment is higher than the rate at which patients leave the sample.

\section{DDD approach}

Based on the prescribed number of DDDs, a considerable proportion of patients discontinue statin treatment during the first year from the first redemption (Figure 1, Table 2). Here, we consider only the first treatment episode for each patient, while in the Appendix we present the same results taking into account all treatment episodes for each patient.

In spite of the relatively long follow-up for most of our patients, survival curves for treatment duration flattened after approximately 800 days for all three DDD-based decision rules. This meant that while the $10^{\text {th }}$ and $25^{\text {th }}$ percentiles could be estimated rather accurately, this was not possible for the median of the distribution (Table 2).

Drug survival was higher among men than women and among older rather than younger individuals (Figure 1). Women and patients under 60 years old in particular have a median survival on their first treatment episode of less than 600 days. For results based on taking into account all treatment episodes for each patient, see Table A1 and Figure A1 in the appendix.

\section{Dose-unit approach}

The estimated treatment duration curve was in general steeper for the dose-unit approach than for the DDD approach (Figure 2, Table 2). While the first decile of treatment duration was larger, the survival curve for treatment duration fell steeply after that and produced the shortest treatment duration estimates of the three methods presented in this paper.

This is clearly appreciated in the estimated duration for the $10^{\text {th }}$ percentile of patients, which is 50 days using the DDD approach ( 1 DDD $=1$ day), and 98 days using the dose-unit approach $(1$ tablet $=1$ day $)$. However, this relationship is eventually reverted. Both the DDD and the dose-unit approaches ( $1 \mathrm{DDD}=1$ tablet $=1$ day) estimate that the duration for the $25^{\text {th }}$ percentile will be 100 days, and once the $50^{\text {th }}$ percentile is reached, the dose unit approach returns an estimated duration of 300 days, while this percentile is not possible to estimate using the DDD approach (Table 2).

Gender and age effects are similar for the dose-unit approach and the DDD approach, albeit with gender effects being less pronounced in this case (Figure 2, Figure A2).

\section{WTD approach}

The reverse WTD approach with random index dates implies selecting a random index date for each patient 
A)

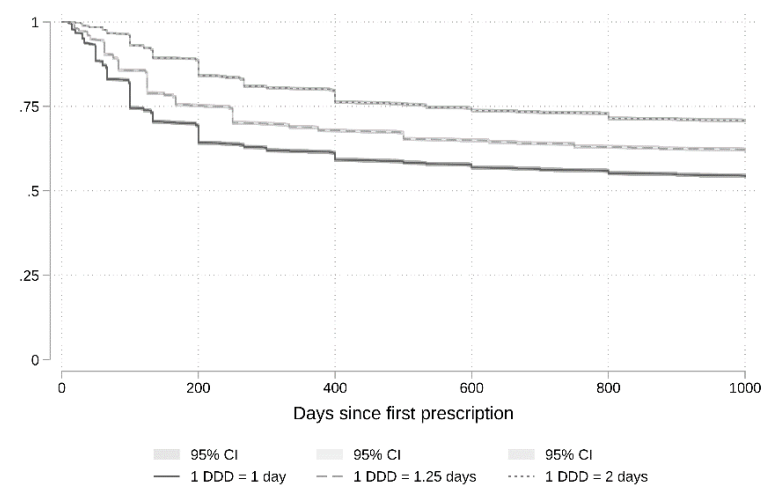

C)

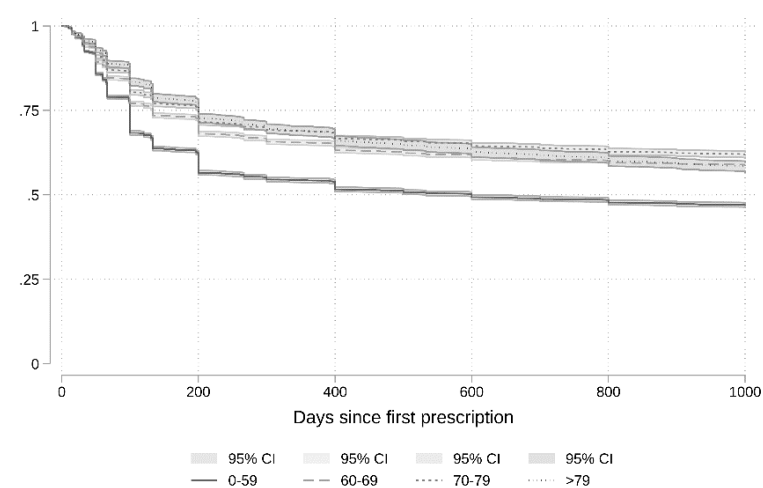

B)

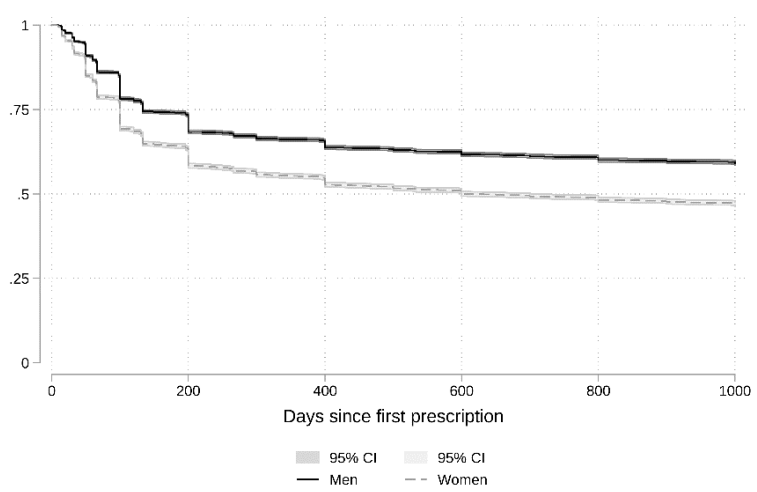

Figure 1. Persistence during first treatment episode based on redeemed number of DDDs. First treatment episode for each patient included only. A) Persistence by number of DDDs consumed/day. B) Persistence by gender (1 DDD = 1 day). C) Persistence by age group (1 DDD = 1 day).

A)

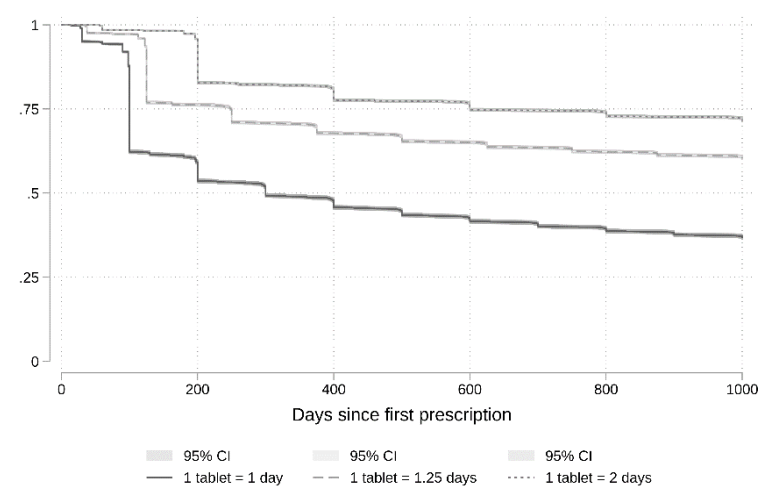

C)

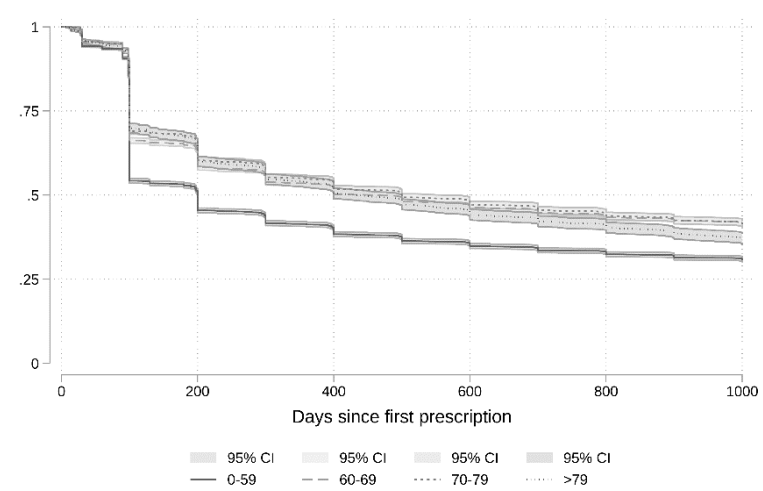

B)

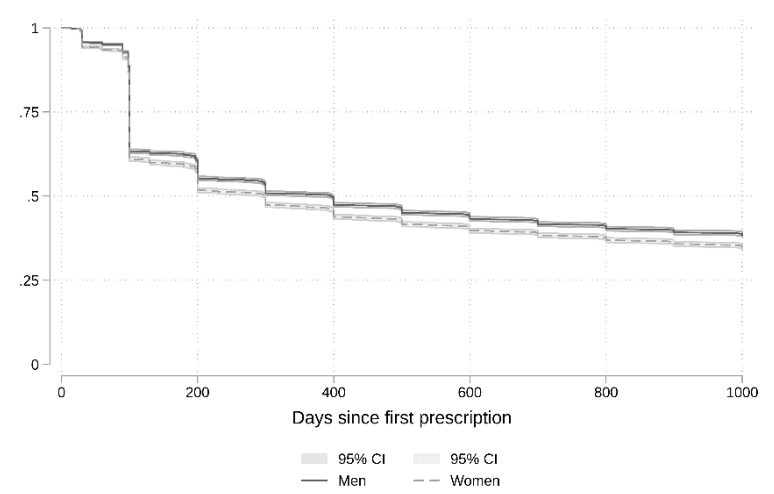

Figure 2. Persistence during first treatment episode based on redeemed number of tablets (dose-unit approach). First treatment episode for each patient included. A) Persistence by number of tablets consumed/day. B) Persistence by gender $(1$ tablet $=1$ day $)$. C) Persistence by age group $(1$ tablet $=1$ day $)$. 
Table 2. Estimated duration of each patient's first treatment episode, DDD, dose-unit and WTD approaches.

\begin{tabular}{lcccc}
\hline & & \multicolumn{3}{c}{ Percentile } \\
\cline { 3 - 5 } Approach & Assumption & 10 th & 25 th & 50th \\
\hline DDD & 1 DDD $=1$ day & $50(50-50)$ & $100(100-100)$ & Not possible to estimate \\
& 1 DDD $=1.25$ days & $75(75-75)$ & $187.5(187.5-245)$ & Not possible to estimate \\
& 1 DDD $=2$ days & $133.33(133.33-133.33)$ & $533.33(533.33-563.33)$ & Not possible to estimate \\
\hline Dose-unit & 1 tablet $=1$ day & $98(98-98)$ & $100(100-100)$ & 300 (300-300) \\
& 1 tablet $=1.25$ days & $125(125-125)$ & $247.5(247.5-250)$ & Not possible to estimate \\
& 1 tablet $=2$ days & $200(200-200)$ & $600(600-660)$ & Not possible to estimate \\
\hline \multirow{2}{*}{ WTD } & No grace period & $151.11(151.11-151.11)$ & $453.34(453.34-453.34)$ & Not possible to estimate \\
& 15 days grace period & $166.11(166.11-166.11)$ & $498.34(498.34-664.45)$ & Not possible to estimate \\
& 30 days grace period & $181.11(181.11-181.11)$ & $724.45(724.45-724.45)$ & Not possible to estimate \\
\hline
\end{tabular}

A)

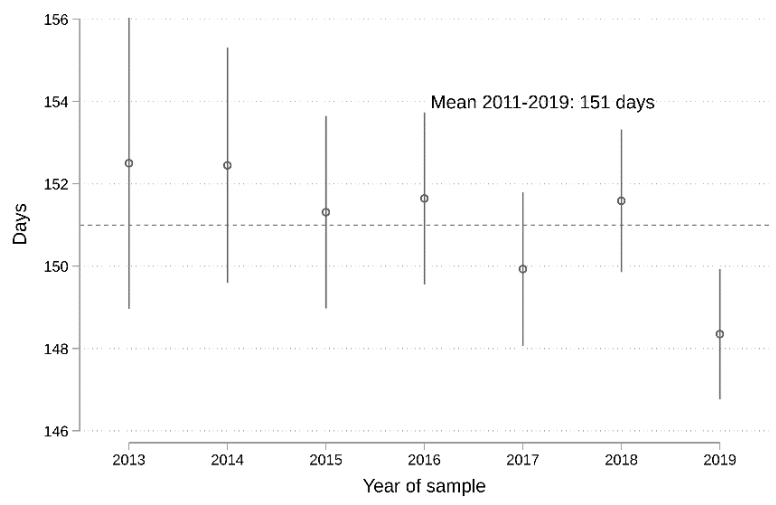

C)

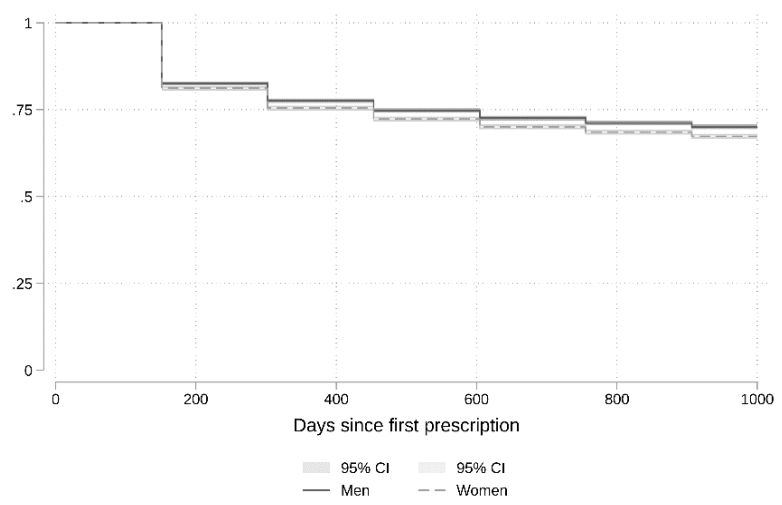

B)
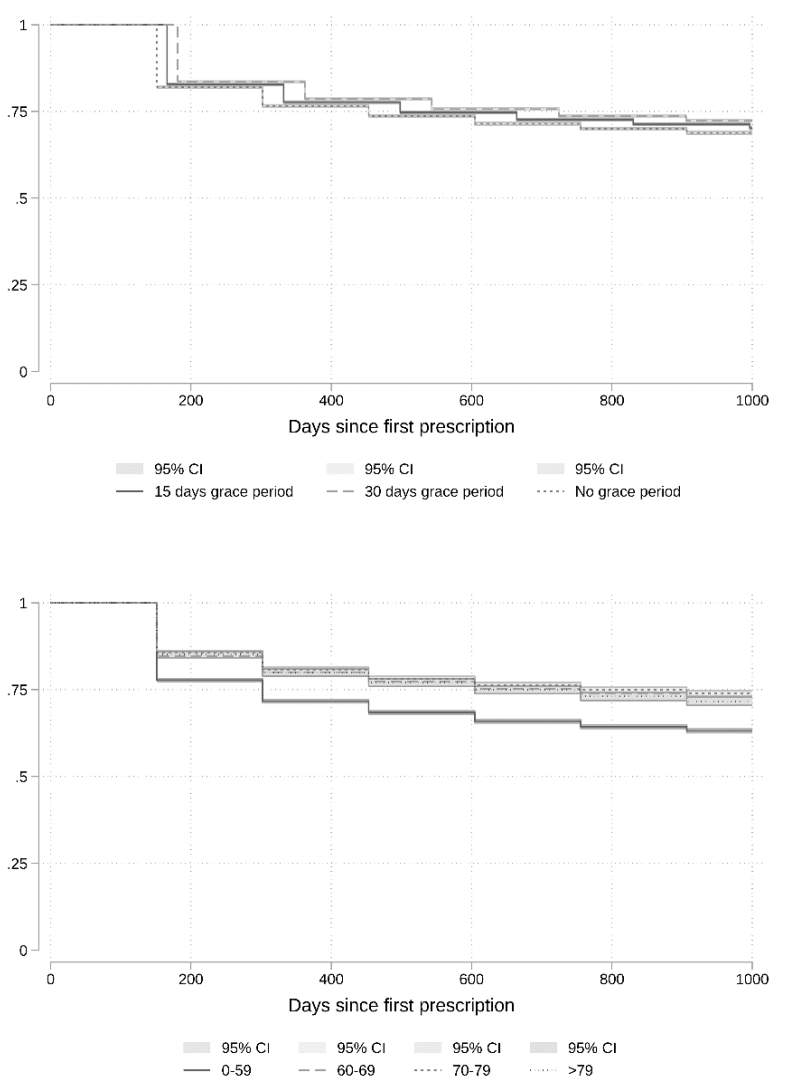

Figure 3. Persistence based on the reverse WTD method with random index dates. First treatment episode for each patient included only. A) Estimated length of prescriptions, in days, based on the $90^{\text {th }}$ percentile, by year of sample. Dashed line indicates the average estimated prescription length over all samples, 151 days. B) Persistence, by grace period. C) Persistence by gender. D) Persistence by age group.

within a sampling window of one year and then calculating the waiting time for each patient from their last previous prescription to the index date. In the appendix, we also present results using a fixed index date, namely the last calendar day for each year.

As described in the Methods section, the first step was to estimate the time elapsed between each patient's last prescription and the randomly sampled index date. Index dates were sampled on a calendar year basis, and previous prescriptions were allowed to be, at most, a year before that. We then estimated the duration for the $90^{\text {th }}$ percentile in our sample (Table 2, Figure 3 ).
The average $90^{\text {th }}$ percentile across the years was 151 days. This was then applied as the duration of each prescription in our dataset, in order to estimate a survival curve for treatment duration. We also included two alternative scenarios in which patients also had a 15and 30-day grace period following each prescription (Figure 3). As in the case of the DDD approach, survival curves flattened out making it impossible to estimate the median treatment duration among patients.

Men had slightly longer treatment duration than women, and younger patients had the shortest treatment courses (Figures 3 and A3). When no grace period was 
applied, the reverse WTD approach resulted in estimates of the $10^{\text {th }}$ percentile of treatment duration that lie between those from the DDD ( $1 \mathrm{DDD}=2$ days) and dose-unit $(1$ tablet $=2$ days $)$ approaches. For the $25^{\text {th }}$ percentile of treatment durations, the WTD estimates are lower than those obtained with these methods. When a grace period was allowed for, the reverse WTD estimated the highest treatment durations of the three methods.

\section{Discussion}

Statin treatment persistence in Norway is less than desirable irrespective of method used to estimate drug survival and treatment duration. When employing simple rules for prescription durations, the estimated treatment duration was overall higher with the DDD than the dose-unit approach (Figures 1 and 2).

However, the method used to estimate treatment duration yields results that vary from reasonable to poor persistence depending on method.

A major strength of this study lies in the national dataset which presumably is almost complete [11]. A small number of patients may occasionally buy their drugs abroad, and some patient data may be entered incorrectly in the pharmacies. The pharmacy computer systems, however, have logical checks for the 11-digit personal identification numbers used in Norway. For quality assurance of the NorPD data, the Norwegian Institute of Public Health conducts routine checks on the data before they are transferred to the NorPD, and a number of queries are carried out monthly or half-yearly to identify possible errors or inconsistencies [11]. A major weakness, however, is that we do not know whether the patients actually take the drugs they redeem at the pharmacies.

Similar to our study, previous studies have found suboptimal persistence on statin treatment, although the level depends on the method used to estimate treatment duration. Larsen and colleagues found low persistence with statins in Bologna, Italy and Funen, Denmark in 1994-6, with the highest persistence in Funen (91\% after two years) [12]. Their findings, however, were based on requiring only one prescription redemption in 1996 among all treated in 1994, and thus they ignored any pauses in treatment during the intervening period. An Australian study found that the Medication Possession Ratio (MPR) depended on subsidy scheme, where MPR was simply determined by assuming a 30-day prescription duration for all [13]. A survey-based study in USA report high rates of discontinuation $(30 \%)$ within one year of starting statin treatment, while a pharmacy claims-based study found that discontinuation was associated with co-payment and exceeded 50\% in less than four years after treatment initiation [14]. A Danish study found that even among patients who have had myocardial infarction, persistence after three years was no more than $80 \%$, although these patients are likely to have the highest benefit from persisting with their statin treatment [15]. That study used a grace period of 90 days and determined prescription duration from average time to next prescription and average amount supplied. Norwegian studies on use of statins based on the NorPD have focused on understanding variation in statin use, either due to regional or educational differences. They could however not do long-term follow-up as we have done in our study $[11,16]$. From a methodological point of view, it is evident that the studies use differing definitions and methods for estimation of prescription duration and dosage. Also, they differ in considering all users versus new users only, duration of wash-out-period, use of grace periods and explicit definition of treatment episodes as a sequence of redeemed prescriptions.

In a review of intermittent non-daily statin dosing, Keating and co-authors identified 10 case-series and controlled studies [17]. The studies indicate that nondaily statin intake may be reasonable for patients with statin side effects. In one randomized placebo-controlled trial of weekly intake of $5-10 \mathrm{mg}$ rosuvastatin, $20 \%$ of the patients achieved their treatment target [18]. Even though there are no clinical end-point studies of nondaily use of statins, it seems plausible to assume that absolute daily intake may not be essential. Against this background, the WTD approach may be the most relevant for studies of statin persistence as we found that it resulted in 10 and 25 percentiles of drug survival that were larger than those based on DDD or dose-unit approach. As the reverse WTD relies on actual dispensing and usage patterns, this could indicate that on average patients use a lower dose than they have been prescribed, possibly due to not taking the medication each day.

The variation in methodology used to estimate persistence and prescription durations, and the subsequent variation in results, is well-known in pharmacoepidemiology, in particular for antidepressant treatment $[19,20]$. The variation makes it difficult to compare results from different studies based on claims or pharmacy data, since results, as we also find here, depend heavily on assumptions made regarding prescription duration, allowance for stockpiling and use of grace periods. Methods have been suggested, which are data-driven in an attempt to overcome this. Two approaches are based on past prescription history as input to complex decision algorithms, the PRE2DUP [21] which has been validated against expert defined treatment status, and the Medicine Macro, which although not yet validated have been compared against simpler approaches [22]. The Waiting Time Distribution approach is also data driven, but is based on a formal statistical model, which allows maximum likelihood estimation and explicit inclusion of covariates in the estimation [10]. The method has been validated for the oral anticoagulant warfarin, a drug with near chronic treatment, albeit with variable intensity [9].

While it is important to identify and offer statins to those who could benefit from therapy, it is even more important to ensure that patients continue to achieve the maximum benefit. Statin persistence should therefore be regularly analysed by clinicians. Here, we conclude that treatment duration and drug survival depend heavily on the methods employed to estimate them. The choice 
of method should therefore be informed by the clinical situation and sensitivity analyses be provided to aid readers in assessing the robustness of the findings. Treatment discontinuation may be fatal for patients on warfarin because poor persistence may cause cerebral stroke. In contrast, the atherosclerotic process is slow, and some days of statin discontinuation may not have a substantial impact on the clinical outcome. Against this background, the WTD approach appears attractive, although it is less intuitive.

\section{Ethics approval}

This study is part of a project approved by the Regional Ethical Committee (reference 153702).

\section{Conflict of interest}

The data collection was financed by Novartis Pharma, Norway. Francisco Oteiza, Christoffer Bugge, Hanna Isabel Høyland, Henrik Støvring and Ivar Sønbø Kristiansen are affiliated with Oslo Economics, which has received project payment from Novartis.

\section{REFERENCES}

1. Mach F, et al. 2019 ESC/EAS Guidelines for the management of dyslipidaemias: lipid modification to reduce cardiovascular risk: The Task Force for the management of dyslipidaemias of the European Society of Cardiology (ESC) and European Atherosclerosis Society (EAS). Eur Heart J 2019; 41(1): 111-88.

2. Deshpande S, et al. A systematic review to assess adherence and persistence with statins. Curr Med Res Opin 2017; 33(4): 769-78.

3. De Vera MA, et al. Impact of statin adherence on cardiovascular disease and mortality outcomes: a systematic review. Br J Clin Pharmacol 2014; 78(4): 684-98.

4. De Geest S, et al. Improving Medication Adherence Research Reporting: European Society for Patient Adherence, Compliance and Persistence Medication Adherence Reporting Guideline. J Cardiovasc Nurs 2019; 34(3): 199-200.

5. Vrijens B, et al. A new taxonomy for describing and defining adherence to medications. Br J Clin Pharmacol 2012; 73(5): 691-705.

6. Cramer JA, et al. Medication compliance and persistence: terminology and definitions. Value Health 2008; 11(1): 44-7.

7. WHO Collaborating Centre for Drug Statistics Methodology. DDD - Definitions and General Considerations. 2018; Available from: https://www.whocc.no/ddd/definition_and_general_considera/.

8. Støvring H, Pottegård A, Hallas J. Estimating medication stopping fraction and real-time prevalence of drug use in pharmaco-epidemiologic databases. An application of the reverse waiting time distribution. Pharmacoepidemiol Drug Saf 2017; 26(8): 909-16.

9. Thrane JM, et al. Empirical validation of the reverse parametric waiting time distribution and standard methods to estimate prescription durations for warfarin. Pharmacoepidemiol Drug Saf 2018; 27(9): 1011-8.

10. Støvring H, Pottegård A, Hallas J. Refining estimates of prescription durations by using observed covariates in pharmacoepidemiological databases: an application of the reverse waiting time distribution. Pharmacoepidemiol Drug Saf 2017; 26(8): 900-8.

11. Furu K. Establishment of the nationwide Norwegian Prescription Database (NorPD) - new opportunities for research in pharmacoepidemiology in Norway. Norsk Epidemiologi 2008; 18(2): 129-36.

12. Larsen J, et al., Lack of adherence to lipid-lowering drug treatment. A comparison of utilization patterns in defined populations in Funen, Denmark and Bologna, Italy. Br J Clin Pharmacol 2000; 49(5): 463-71.

13. Warren JR, et al. Factors influencing adherence in long-term use of statins. Pharmacoepidemiol Drug Saf 2013; 22(12): 1298-307.

14. Ellis JJ, et al. Suboptimal statin adherence and discontinuation in primary and secondary prevention populations. J Gen Intern Med 2004; 19(6): 638-45.

15. Gislason GH, et al., Long-term compliance with beta-blockers, angiotensin-converting enzyme inhibitors, and statins after acute myocardial infarction. Eur Heart $J$ 2006; 27(10): 1153-8.

16. Selmer R, et al. Statin treatment in a cohort of 20212 men and women in Norway according to cardiovascular risk factors and level of education. Br J Clin Pharmacol 2009; 67(3): 355-62.

17. Keating AJ, Campbell KB, Guyton JR. Intermittent nondaily dosing strategies in patients with previous statininduced myopathy. Ann Pharmacother 2013; 47(3): 398-404.

18. Kennedy SP, et al. Efficacy and tolerability of once-weekly rosuvastatin in patients with previous statin intolerance. J Clin Lipidol 2011; 5(4): 308-15.

19. Gardarsdottir $\mathrm{H}$, et al. Construction of drug treatment episodes from drug-dispensing histories is influenced by the gap length. $J$ Clin Epidemiol 2010; 63(4): 422-7.

20. Gardarsdottir H, Heerdink ER, Egberts AC. Potential bias in pharmacoepidemiological studies due to the length of the drug free period: a study on antidepressant drug use in adults in the Netherlands. Pharmacoepidemiol Drug Saf 2006; 15(5): 338-43. 
21. Tanskanen A, et al. From prescription drug purchases to drug use periods - a second generation method (PRE2DUP). BMC Med Inform Decis Mak 2015; 15: 21.

22. medicinMacro: Medicin macro to estimate prescription lengths and averages 2021; Available from: https://rdrr.io/github/tagteam/heaven/man/medicinMacro.html.

Table A1. Estimated duration of all treatment episodes.

\begin{tabular}{lcccc}
\hline & & \multicolumn{3}{c}{ Percentile } \\
\cline { 3 - 5 } Approach & Assumption & 10 th & 25 th & 50 th \\
\hline DDD & 1 DDD $=1$ day & $49(49-49)$ & $100(100-100)$ & $666.66(600-733.33)$ \\
& 1 DDD $=1.25$ days & $62.5(62.5-62.5)$ & $125(125-125)$ & Not possible to estimate \\
& 1 DDD $=2$ days & $120(120-120)$ & $400(400-400)$ & Not possible to estimate \\
\hline Dose-unit & 1 tablet $=1$ day & $90(90-90)$ & $100(100-100)$ & $200(200-200)$ \\
& 1 tablet $=1.25$ days & $125(125-125)$ & $125(125-125)$ & $\left.2610(2,460-N)^{*}\right)$ \\
& 1 tablet $=2$ days & $200(200-200)$ & $592(556-600)$ & Not possible to estimate \\
\hline WTD & No grace period & $131.91(131.91-131.91)$ & $263.82(263.82-263.82)$ & Not possible to estimate \\
& 15 days grace period & $146.91(146.91-146.91)$ & $440.72(440.72-440.72)$ & Not possible to estimate \\
& 30 days grace period & $161.91(161.91-161.91)$ & $485.72(485.72-485.72)$ & Not possible to estimate \\
\hline
\end{tabular}

*NE $=$ Not possible to estimate.

A)

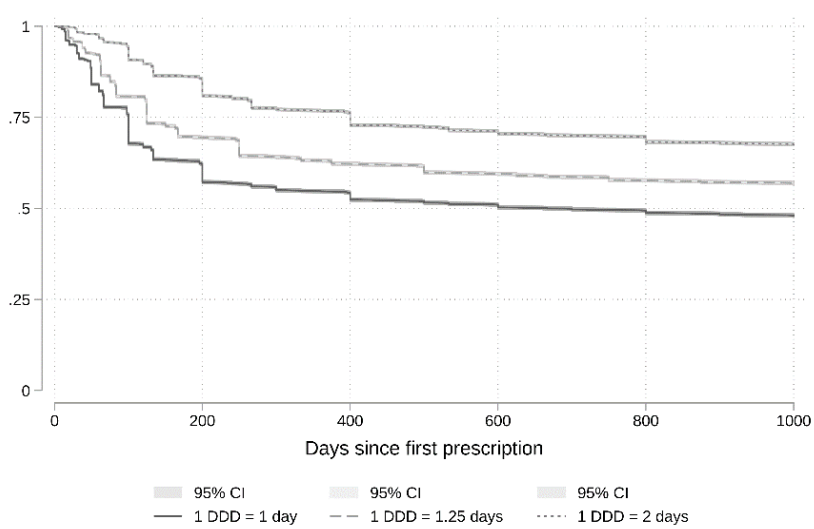

C)

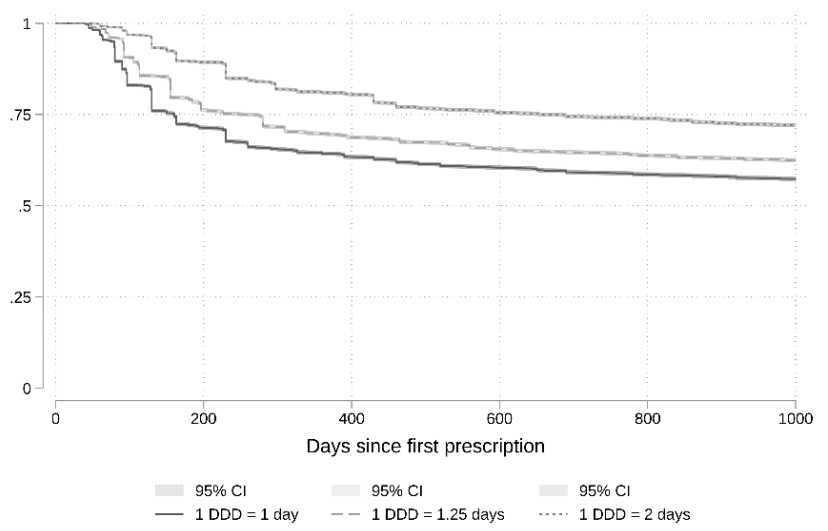

B)

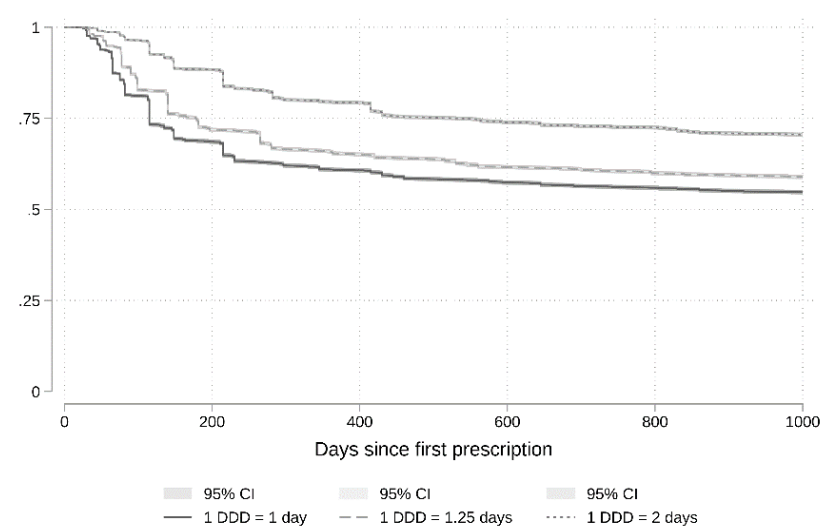

Figure A1. Persistence based on redeemed number of DDDs assuming different number of DDDs consumed per day. Legend: All treatment episodes for each patient included. A) Persistence assuming no grace period. B) Assuming a 15-day grace period at the end of each prescription. C) Assuming a 30-day grace period at the end of each prescription. 
A)

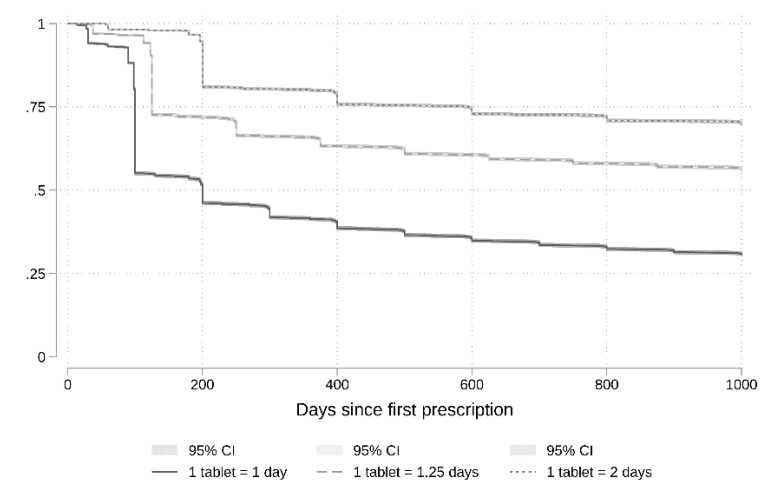

C)

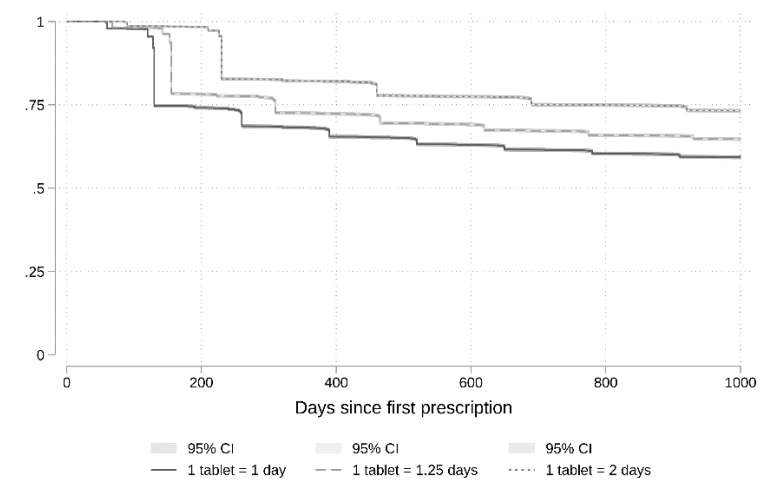

B)

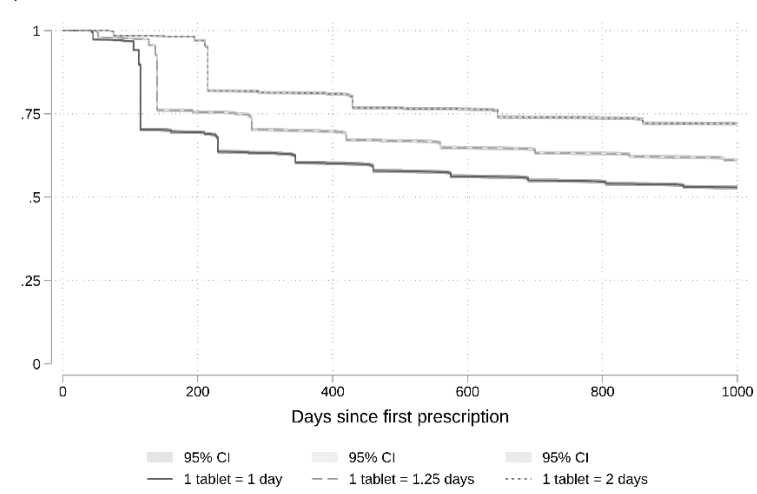

Figure A2. Persistence based on redeemed number of tablets assuming different number of tablets consumed per day. Legend: All treatment episodes for each patient included. A) Persistence assuming no grace period. B) Assuming a 15-day grace period at the end of each prescription. C) Assuming a 30-day grace period at the end of each prescription.

A)

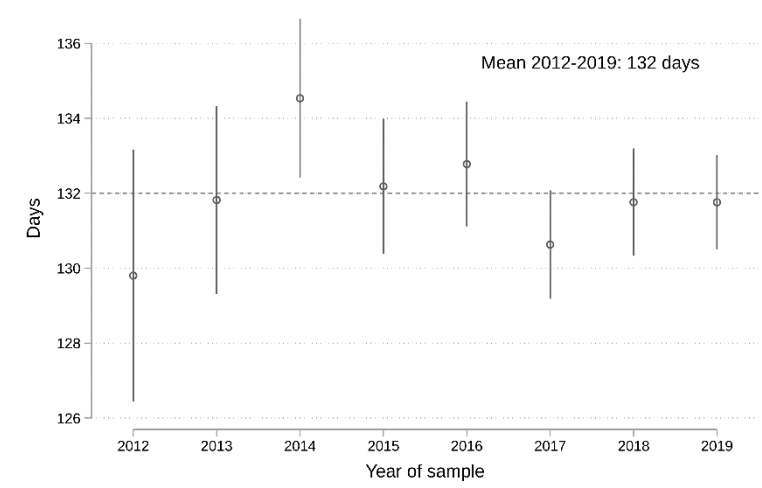

C)

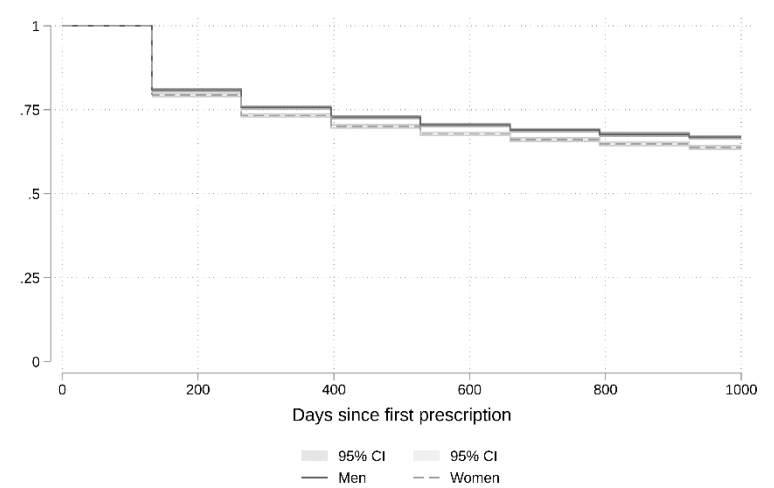

B)

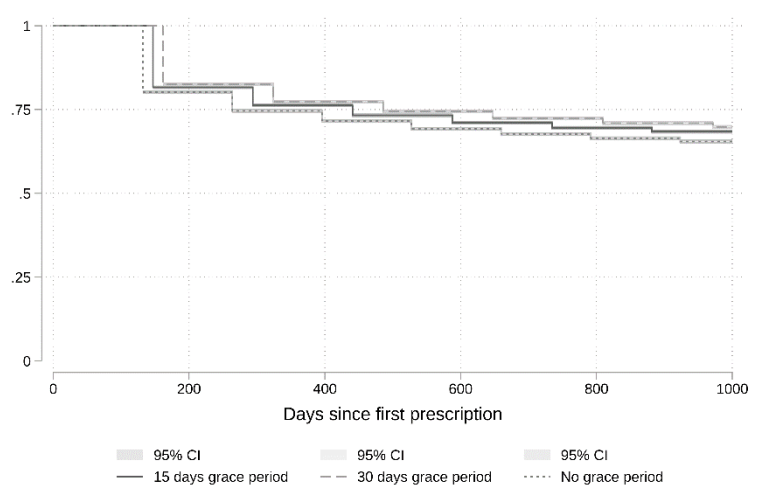

D)

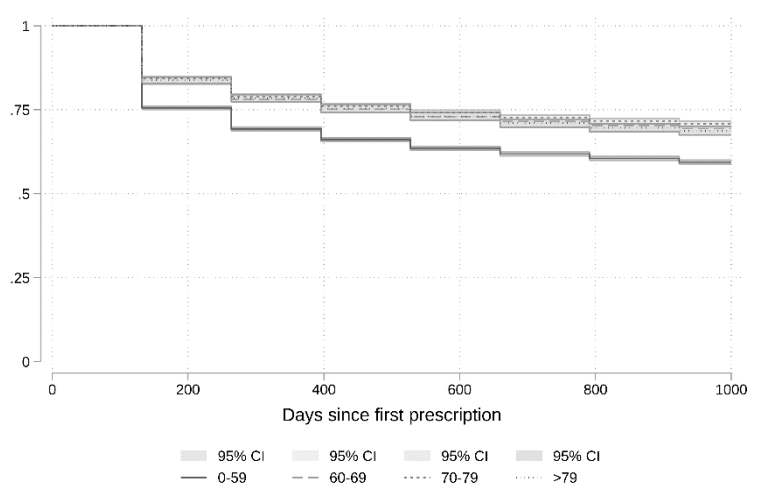

Figure A3. Persistence based on the reverse WTD method taking the end of each calendar year as fixed index date. Legend: First treatment episode for each patient only. A) Estimated length of prescriptions, in days, based on the 90th percentile, by year of sample. B) Persistence, by grace period. C) Persistence by gender. D) Persistence by age group. 